\title{
The N-Terminal Domain of Human DNA Helicase Rtel1 Contains a Redox Active Iron-Sulfur Cluster
}

\author{
Aaron P. Landry and Huangen Ding \\ Department of Biological Sciences, Louisiana State University, 202 Life Sciences Building, Baton Rouge, LA 70803, USA
}

Correspondence should be addressed to Huangen Ding; hding@lsu.edu

Received 9 June 2014; Accepted 8 July 2014; Published 24 July 2014

Academic Editor: Cheng-Yang Huang

Copyright (C) 2014 A. P. Landry and H. Ding. This is an open access article distributed under the Creative Commons Attribution License, which permits unrestricted use, distribution, and reproduction in any medium, provided the original work is properly cited.

\begin{abstract}
Human telomere length regulator Rtell is a superfamily II DNA helicase and is essential for maintaining proper length of telomeres in chromosomes. Here we report that the N-terminal domain of human Rtell (RtelN) expressed in Escherichia coli cells produces a protein that contains a redox active iron-sulfur cluster with the redox midpoint potential of $-248 \pm 10 \mathrm{mV}$ ( $\mathrm{pH} 8.0$ ). The iron-sulfur cluster in RtelN is sensitive to hydrogen peroxide and nitric oxide, indicating that reactive oxygen/nitrogen species may modulate the DNA helicase activity of Rtell via modification of its iron-sulfur cluster. Purified RtelN retains a weak binding affinity for the single-stranded (ss) and double-stranded (ds) DNA in vitro. However, modification of the iron-sulfur cluster by hydrogen peroxide or nitric oxide does not significantly affect the DNA binding activity of RtelN, suggesting that the iron-sulfur cluster is not directly involved in the DNA interaction in the N-terminal domain of Rtell.
\end{abstract}

\section{Introduction}

In vertebrates, telomeres are the protective structures at the end of chromosomes and are composed of a repetitive TTAGGG sequence and associated proteins that form a core structure known as the Shelterin complex $[1,2]$. Telomeres become shorter with each round of DNA replication and are compensated for by telomerase [3]. In addition to telomerase, the telomere length is regulated by other genetic [4] and epigenetic [5] factors. Among them, telomere length regulator 1 (Rtell) has an essential role in maintaining proper length of telomeres $[4,6]$. Deletion of Rtell in mice is embryonic lethal with increased incidence of chromosomal abnormalities and telomere loss [4]. In humans, Rtell is highly expressed in several types of tumor tissues [7] and specific mutations in Rtell have been attributed to dyskeratosis congenita and Hoyeraal-Hreidarsson syndrome $[8,9]$.

Human Rtell is a superfamily II DNA helicase [10] and is homologous to other human DNA helicases XPD (Xeroderma pigmentosum factor D) [11], FancJ (Fanconi's anaemia complementation group J)/BACH1 (for BRCA1-associated Cterminal helicase) [12, 13], and ChlR1 (a protein required for normal mitotic progression) [14]. However, unlike other DNA helicases, Rtell preferentially disrupts the D-loop of the T-loop structure formed at the end of telomeres [15] and may act as an antirecombinase to prevent formation of $\mathrm{D}$ loop $[6,16]$. In the absence of Rtell, the T-loop structure could be erroneously resolved as a substrate for homologous recombination [16], leading to telomere deficiency. On the other hand, excessive activity of Rtell would be detrimental as increased Rtell helicase activity would disengage the Tloop structure, leading to telomere deprotection and genomic instability [16]. Thus, the helicase activity of Rtell must be tightly regulated to maintain proper length of telomeres in chromosomes [6].

The sequence alignment analyses revealed that the $\mathrm{N}$ terminal domain of human Rtell contains a conserved region for hosting a putative iron-sulfur cluster via four cysteine residues (Figure 1) [17]. It has previously been reported that the DNA helicase Rad3 from yeast [18], XPD homologues from archaea [19-22], DNA-damage-inducible DNA helicase DinG from Escherichia coli [23], and AddAB-type helicasenuclease from Bacillus subtilis [24] contain a [4Fe-4S] cluster essential for the helicase activity. However, the existence of 


$\begin{array}{lcll}\text { Rad3 } & 108 & \text { LTSRKNLCLHPEVSKERKGTVVDEKCRRMTNGQAKRKLEEDPeA } & \text { NVELCEYHENLY } \\ \text { XPD } & 109 & \text { LSSRKNLCIHPEVTPLRFGKDVDGKCHSLTASYVRAQYQHD--T } & \text { SLPHCRFYEEFD } \\ \text { Rtel1 } & 162 & \text { LGSREQLCIHPEVKKQESNHLQIHLCRKKVASR----------- } & -- \text { SCHFYNNVE } \\ \text { FancJ } & 276 & \text { LSSRDHTCVHPEVVG---NFNRNEKCMELLDGKNGK-------- } & --- \text { SCYFYHGVHKID } \\ \text { Chl1 } & 260 & \text { LGSRQNLCVNEDVKSLGSVQLINDRCVDMQRSRHEKKKGAEEEK [7] KQAACPFYNHEQ } \\ & & \\ \text { RAD3 } & 182 & \text { LKYCEEKTLCPYFIVRRMISLCNIIIYSYHYLLDPKIAERVSNEVSKDSIVIFDEAHN } \\ \text { XPD } & 181 & \text { KALGRRQGWCPYFLARYSILHANVVVYSYHYLLDPKIADLVSKELARKAVVVFDEAHN } \\ \text { Rtel1 } & 222 & \text { VKSGSKHRVCPYLSRNLKQQADIIFMPYNYLLDAKSRRAHNIDL-KGTVVIFDEAHN } \\ \text { FancJ } & 341 & \text { VSLGKKLKACPYYTARELIQDADIIFCPYNYLLDAQIRESMDLNL-KEQVVILDEAHN } \\ \text { Chl1 } & 341 & \text { LALGKEARACPYYGSRLAIPAAQLVVLPYQMLLHAATRQAAGIRL-QDQVVIIDEAHN }\end{array}$

FIGURE 1: Alignment of the N-terminal domain of some human DNA helicases. Sequence alignment of yeast DNA helicase Rad3 and human DNA helicase XPD, Rtel1, FancJ, and Chl1 was generated by cobalt (NCBI). Four conserved cysteine residues proposed for hosting an ironsulfur cluster in these DNA helicases are highlighted in yellow.

the iron-sulfur cluster in any human DNA helicases has not been experimentally demonstrated. Here, we report that expression of the N-terminal domain (residues 1-312) of human Rtell (RtelN) in E. coli cells produces a protein that contains a redox active iron-sulfur cluster with redox midpoint potential $\left(E_{m 7}\right)$ of $-248 \pm 10 \mathrm{mV}(\mathrm{pH} 8.0)$. Purified RtelN retains a weak binding activity for the single-stranded (ss) and double-stranded (ds) DNA, and disruption of the iron-sulfur cluster by hydrogen peroxide or nitric oxide does not affect the DNA binding activity of RtelN, suggesting that iron-sulfur cluster in the N-terminal domain may not be directly involved in the DNA interaction in Rtell.

\section{Materials and Methods}

2.1. Protein Preparation. The DNA fragment encoding the Nterminal domain (residues 1-312) (RtelN) of human regulator of telomere length 1 (Rtel1) was synthesized for expression in $E$. coli cells (Genescript co.). The gene was subcloned into an expression plasmid $\mathrm{pET}^{2} 8 \mathrm{~b}^{+}$which was introduced into E. coli BL21 cells. The E. coli cells hosting the expression plasmid were grown in $\mathrm{LB}$ media to an $\mathrm{OD}_{600 \mathrm{~nm}}$ of $\sim 0.6$ before isopropyl $\beta$-D-1-thiogalactopyranoside $(200 \mu \mathrm{M})$ was added to induce the protein expression for three hours. The cells were harvested and passed through French press once. Recombinant RtelN in pellets was solubilized by adding urea $(6 \mathrm{M})$, and the protein was purified using a nickel-agarose column attached to a FPLC system (GE Biosciences), followed by passing through a HiTrap desalting column. The molecular weight of RtelN was confirmed by the MALDI mass spectrometer (Chemistry Department, LSU). The concentration of purified RtelN was measured from the absorption peak at $280 \mathrm{~nm}$ using an extinction coefficient of $25.5 \mathrm{mM}^{-1} \mathrm{~cm}^{-1}$. The total iron content in purified RtelN sample was determined using an iron indicator FerroZine [25]. The total acidlabile sulfide content was determined according to Siegel's method [26]. The single-stranded DNA binding protein SSB [27] was prepared as described previously [28].

2.2. The DNA Binding Activity Assay of RtelN. The DNA binding activity assay was carried out using a fluorescence labeled
40 mer $\left(5^{\prime}-\mathrm{F}^{*}\right.$-AATTGC-GATCTAGCTCGCCAGU-AGCGACCTTATCTGATGA-3'). For single-stranded (ss) DNA binding assay, the 40 mer $(0.5 \mu \mathrm{M})$ was incubated with increasing concentrations of protein in buffer containing Tris (20 mM, pH 8.0), $\mathrm{NaCl}$ (50 mM), $\beta$-mercaptoethanol (1 mM), $\mathrm{MgCl}_{2}(1 \mathrm{mM})$, and bovine serum albumin $(0.5 \mathrm{mg} / \mathrm{mL})$. For double-strand (ds) DNA binding assay, the fluorescence labeled 40 mer was annealed to a complementary ssDNA in an annealing buffer containing Tris $(50 \mathrm{mM}, \mathrm{pH} 8.0), \mathrm{NaCl}$ (50 $\mathrm{mM})$, and $\mathrm{MgCl}_{2}(10 \mathrm{mM})$. Prepared dsDNA labeled with fluorescence was incubated with increasing concentrations of protein in buffer as described above. After incubation at room temperature for $15 \mathrm{~min}$, samples were loaded on to a $0.6 \%$ agarose gel in TAE buffer. The agarose gel was run at $10 \mathrm{~V}$ per $\mathrm{cm}$ for $30 \mathrm{~min}$ at room temperature and photographed in a KODAK Gel Logic 200 Imaging System.

2.3. Redox Titration of the RtelN Iron-Sulfur Cluster. A specially-designed cuvette was used for redox titration experiments as described by Leslie Dutton [29]. Briefly, purified RtelN $(20 \mu \mathrm{M})$ dissolved in buffer containing Tris (50 mM, pH 8.0) and $\mathrm{NaCl}(500 \mathrm{mM})$ was incubated with a redox mediator safranin $\mathrm{O}(1 \mu \mathrm{M})$ in a sealed cuvette and equilibrated with pure argon gas for 45 minutes at room temperature. The redox potential was adjusted by adding a small amount of freshly prepared sodium dithionite using a gas-tight $10-\mu \mathrm{L}$ Hamilton micro-syringe (Hamilton Co., Reno, NV). The redox potential was monitored with a redox microelectrode (Microelectrodes Inc., Bedford, $\mathrm{NH}$ ) which was calibrated using a standard ZoBell solution $\left(E_{h}\right.$ $=+238 \mathrm{mV})$ containing potassium ferricyanide $(5 \mathrm{mM})$ and potassium ferrocyanide $(5 \mathrm{mM})$ in buffer containing Tris (20 mM, pH 8.0) and $\mathrm{NaCl}(500 \mathrm{mM})$. The redox titration data were fitted to the Nernst equation with $n=1$ using KaleidaGraph (Synergy Software co.).

2.4. Hydrogen Peroxide and Nitric Oxide Treatments of RtelN. For hydrogen peroxide $\left(\mathrm{H}_{2} \mathrm{O}_{2}\right)$ treatments, purified RtelN was incubated with different concentrations of $\mathrm{H}_{2} \mathrm{O}_{2}$ at room temperature for $30 \mathrm{~min}$, followed by repurification of the protein from the incubation solutions. For nitric oxide (NO) treatments, purified RtelN dissolved in a sealed 
vial was purged with pure argon gas for $15 \mathrm{~min}$, followed by incubation with the NO-releasing reagent diethylamine NONOate (Cayman Chemicals co.) at $37^{\circ} \mathrm{C}$ for $10 \mathrm{~min}$. RtelN was repurified after the NO treatment. Modification of the iron-sulfur cluster in RtelN by $\mathrm{H}_{2} \mathrm{O}_{2}$ or $\mathrm{NO}$ was quantified by the UV-visible absorption spectrometer.

2.5. The Circular Dichroism (CD) and Electron Paramagnetic Resonance (EPR) Measurements. The circular dichroism (CD) spectra were recorded on a Jasco J-815 CD spectrometer (AgCenter Biotechnology Laboratories, LSU) at room temperature. The composition of secondary structures was obtained using the CDNN program [30]. The electron paramagnetic resonance (EPR) spectra were recorded at X-band on a Bruker ESR-300 spectrometer equipped with an Oxford Instruments 910 continuous flow cryostat. EPR conditions were as follows: microwave frequency, $9.45 \mathrm{GHz}$; microwave power, $10 \mathrm{~mW}$; modulation frequency, $100 \mathrm{kHz}$; modulation amplitude, $2 \mathrm{mT}$; sample temperature, $10 \mathrm{~K}$; receive gain, $1 \times$ $10^{5}$.

\section{Results}

3.1. The N-Terminal Domain of Human Rtell Hosts an IronSulfur Cluster. When the N-terminal domain of human Rtell (RtelN) was expressed in E. coli cells, the cell pellets had a dark-red color (Figure 2(a) insert). Recombinant RtelN was purified from the E. coli cells as described in the Materials and Methods. The UV-visible absorption measurements showed that purified RtelN had an absorption peak at $415 \mathrm{~nm}$ (Figure 2(a)), similar to that of $S$. acidocaldarius XPD [4Fe4S] cluster [22] and E. coli DinG [4Fe-4S] cluster [23]. Purified RtelN was further subjected to the Circular dichroism (CD) measurements. As shown in Figure 2(b), purified RtelN adopted an ordered structure with about $25 \%$ alpha-helix, $32 \%$ beta-sheet, $20 \%$ beta turns, and $22 \%$ random coil. The iron and sulfide content analyses revealed that purified RtelN contained $0.83 \pm 0.13$ iron and $0.75 \pm 0.16$ acid-labile sulfide per protein.

The low iron and sulfide contents in purified RtelN could be due to the protein purification process under denaturation conditions. To fully reconstitute the iron-sulfur clusters in RtelN, renatured protein was reconstituted with excess iron and sulfide as described previously [23]. After reconstitution, the iron and sulfide contents in RtelN were increased to $3.5 \pm 0.3$ iron and $3.2 \pm 0.5$ sulfide per RtelN, respectively, indicating that each RtelN monomer may bind a [4Fe-4S] cluster.

3.2. The Iron-Sulfur Cluster in RtelN Is Redox Active. When freshly prepared sodium dithionite was added to the solution containing RtelN, the absorption peak at $415 \mathrm{~nm}$ of the RtelN iron-sulfur cluster was completely eliminated (Figure 3(a)). The absorption peak at $415 \mathrm{~nm}$ was restored when the reduced RtelN iron-sulfur cluster was reoxidized by oxygen (data not shown), suggesting that the RtelN iron-sulfur cluster can be reversibly reduced. This notion was further confirmed by the electron paramagnetic resonance (EPR) measurements: while purified RtelN was EPR silent, addition of sodium dithionite to purified RtelN produced an EPR spectrum with $g_{x}=1.918$, $g_{y}=1.994$, and $g_{z}=2.050$ (Figure 3(b)), a spectrum similar to that of the reduced E. coli DNA helicase DinG [4Fe4S] cluster [23].

Redox titration experiments were carried out to determine the redox midpoint potential $\left(E_{m}\right)$ of the RtelN ironsulfur cluster. The amplitude of the absorption peak at $415 \mathrm{~nm}$ of RtelN was plotted as a function of redox potentials in the solution (Figure 3(c)). The data from three sets of experiments were fitted to a Nernst equation $(n=1)$ with an $E_{m 8}$ of $-248 \pm 10 \mathrm{mV}$, which is about $140 \mathrm{mV}$ higher than that of the E. coli DinG [4Fe-4S] cluster [23].

3.3. Purified RtelN Has a Weak DNA Binding Activity. The N-terminal domain of the archaeal DNA helicase XPD comprises part of the catalytic center [20]. To test whether the $\mathrm{N}$-terminal domain of Rtell also contributes to the catalytic site, we examined the DNA binding activity of purified RtelN. As shown in Figure 4(a), purified RtelN formed a proteinDNA complex with the single-stranded (ss) DNA. FhuF, an iron-sulfur protein with a similar molecular weight as RtelN but with no known DNA binding activity [31], failed to bind any ssDNA, indicating that the ssDNA binding in RtelN is specific. Nevertheless, compared with the single-stranded DNA binding protein SSB [27], the binding affinity of RtelN for ssDNA was at least 10-fold-weaker. In parallel, we also determined the double-stranded (ds) DNA binding activity of RtelN under the same experimental conditions. Figure 4(b) shows that RtelN could also bind dsDNA with the similar binding affinity as for ssDNA. In contrast, both FhuF and SSB did not bind any dsDNA as expected. Thus, purified RtelN has a binding activity for both ssDNA and dsDNA in vitro.

3.4. The Iron-Sulfur Cluster Is Not Required for the DNA Binding Activity of RtelN. Ironically, iron-sulfur clusters in proteins are often sensitive to reactive oxygen species [32] and nitrogen species $[33,34]$. To test if the iron-sulfur clusters in RtelN can be modified by reactive oxygen species, we incubated RtelN with hydrogen peroxide at room temperature. Figure 5(a) shows that addition of increasing amounts of hydrogen peroxide removed the absorption peak at $415 \mathrm{~nm}$ of purified RtelN, indicating that the iron-sulfur cluster in RtelN are disrupted by hydrogen peroxide. Purified RtelN was also incubated with the nitric oxide-releasing reagent NONOate in solution. Again, the absorption peak at $415 \mathrm{~nm}$ of the RtelN iron-sulfur cluster was largely abolished as the concentration of nitric oxide was increased (Figure 5(b)). Thus, the ironsulfur cluster in RtelN is sensitive to both hydrogen peroxide and nitric oxide.

We then examined the DNA binding activity of RtelN after the protein was treated with hydrogen peroxide and nitric oxide. Figure 5(c) shows that the DNA binding activity of RtelN remained almost the same when the iron-sulfur cluster was modified by hydrogen peroxide or nitric oxide, suggesting that iron-sulfur cluster is not required for the DNA binding activity of RtelN. 


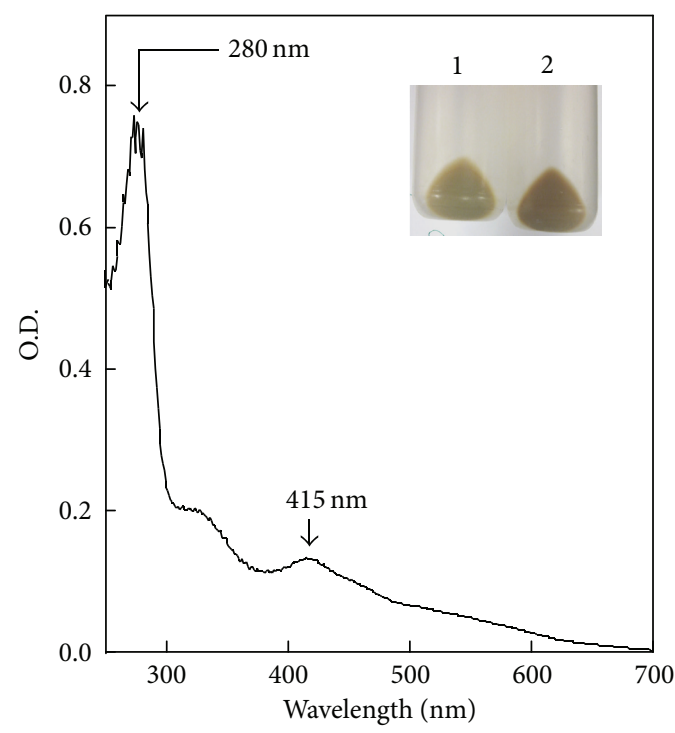

(a)

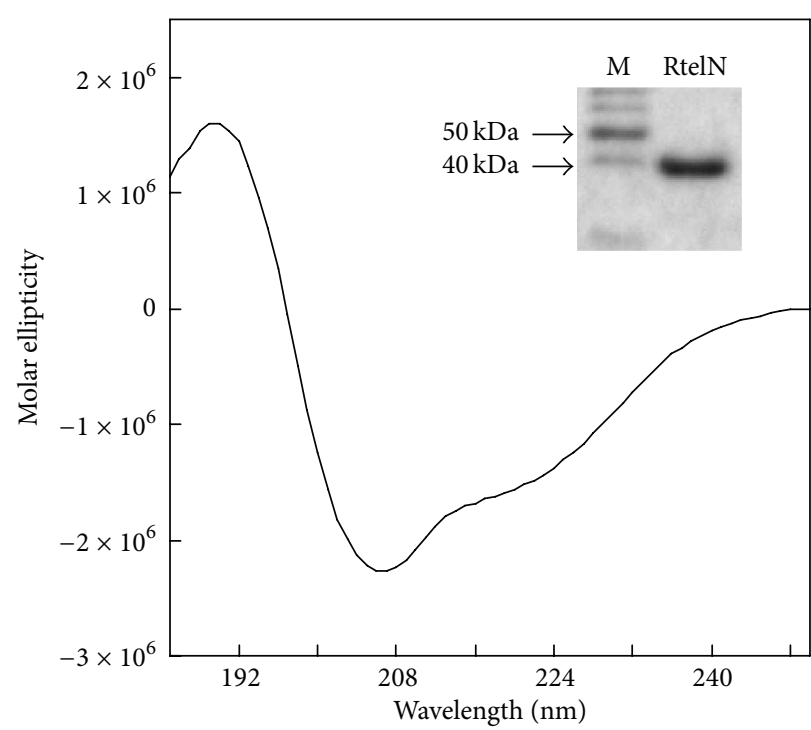

(b)

FIGURE 2: N-terminal domain of Rtell (RtelN) contains an iron-sulfur cluster. (a) UV-visible absorption spectrum of purified RtelN. Purified RtelN $(30 \mu \mathrm{M})$ was dissolved in buffer containing Tris $(20 \mathrm{mM}, \mathrm{pH} 8.0)$ and $\mathrm{NaCl}(500 \mathrm{mM})$. The absorption peak at $415 \mathrm{~nm}$ indicates an iron-sulfur cluster in RtelN. Inset is a photograph of cell pellets after induction with (sample 2) or without (sample 1) IPTG (200 $\mu \mathrm{M}$ ). (b) Circular dichroism (CD) spectrum of purified RtelN. Purified RtelN $(6.75 \mu \mathrm{M})$ was dissolved in potassium phosphate buffer (10 mM, pH 8.0). The spectrum was an average of three scans. Insert is a photograph of the SDS-PAGE gel of purified RtelN. Left lane, molecular marker (M); right lane, purified RtelN.

\section{Discussion}

Recent studies have identified a new set of iron-sulfur clustercontaining enzymes that are involved in DNA processing in bacteria and eukaryotic cells [17]. Among these enzymes are a group of DNA helicases that require an intact ironsulfur cluster for the DNA helicase activity [11, 35]. For example, it has been shown that the DNA helicase Rad3 from yeast and XPD homologues from archaea contain a [4Fe-4S] cluster essential for the enzyme activity [19-22]. In human XPD, mutations in the N-terminal domain that hosts a putative iron-sulfur cluster have been associated with several genetic diseases including xeroderma pigmentosum [20]. Interestingly, in addition to XPD, humans have at least three other DNA helicases: FancJ (Fanconi's anaemia complementation group J)/BACH1 (for BRCA1-associated Cterminal helicase) $[12,13]$, ChlR1 (a protein required for normal mitotic progression) [14], and Rtell of telomere length regulation $[4,6]$ that contain a putative iron-sulfur cluster binding site in the N-terminal domain (Figure 1). However, the existence of the [4Fe-4S] clusters in any of these human DNA helicases has not been experimentally demonstrated. Here we find that the N-terminal domain of human Rtell (RtelN) expressed in E. coli cells contains a redox active [4Fe$4 \mathrm{~S}$ ] cluster and that the iron-sulfur cluster in purified RtelN is highly sensitive to hydrogen peroxide and nitric oxide. The results suggest that human Rtell, like XPD from archaea [1922], likely contains a [4Fe-4S] cluster.

Despite the findings of the iron-sulfur clusters in these DNA helicases, specific function of the [4Fe-4S] clusters in the DNA helicases remains largely elusive [17]. In previous studies, we reported that oxidation of the reduced iron-sulfur cluster in E. coli DNA helicase DinG reversibly switches on the enzyme activity, and proposed that iron-sulfur cluster may regulate the helicase activity in response to redox signals [23]. Here, we have tested the idea further in the human DNA helicase Rtell. While attempts to purify a full-length human Rtell from E. coli cells were not successful, we were able to prepare the soluble $\mathrm{N}$-terminal domain (residues 1-312) of human Rtell (RtelN). The results demonstrated that RtelN contains a redox active iron-sulfur cluster with a redox midpoint potential of $-248 \pm 10 \mathrm{mV}$ ( $\mathrm{pH} \mathrm{8.0)}$ (Figure 3). The redox potential in cytosol and nucleus of mammalian cells has been reported to be around $-325 \mathrm{mV}(\mathrm{pH} 7.0)$ [36]. However, when cells are under oxidative stress or during apoptosis and differentiation, the intracellular redox potential could increase to as high as $+200 \mathrm{mV}$ [37]. Assuming the redox midpoint potential of the iron-sulfur cluster in Rtell is similar to that in RtelN, we would expect that the ironsulfur cluster in Rtell is in reduced state in cells under normal physiological conditions. Under oxidative stress or during apoptosis and differentiation [37], the iron-sulfur cluster in Rtell would be fully oxidized. We envision that oxidation of the reduced iron-sulfur cluster in Rtell, like that in the E. coli DinG [23], may change the DNA helicase activity of the protein in response to redox signals. Thus, fluctuation of intracellular redox potential may result in change of the Rtell's DNA helicase activity by changing the redox state of the ironsulfur cluster. While the iron-sulfur cluster appears to be dispensable for the DNA binding activity of purified RtelN 


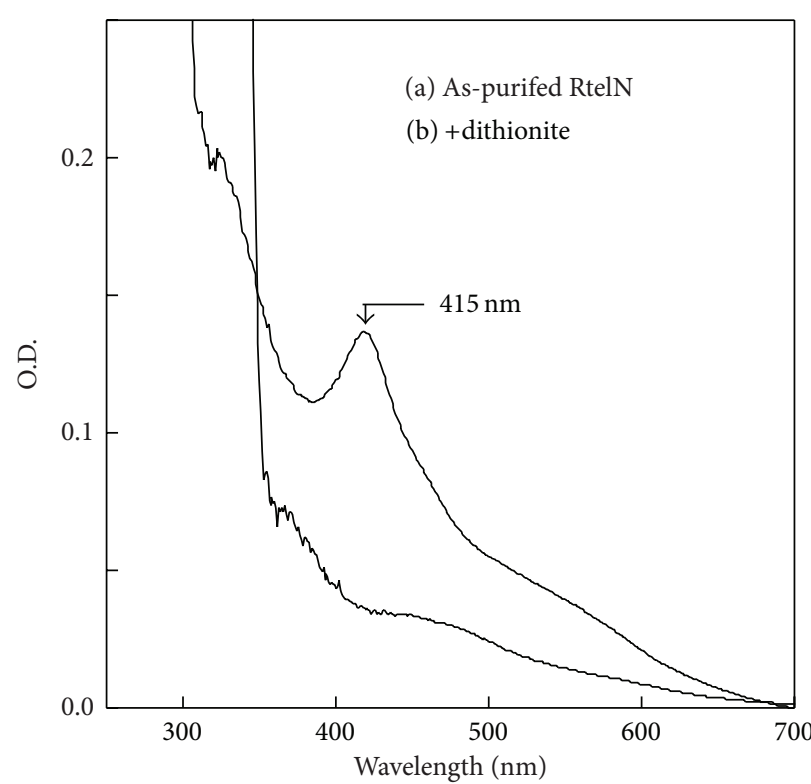

(a)
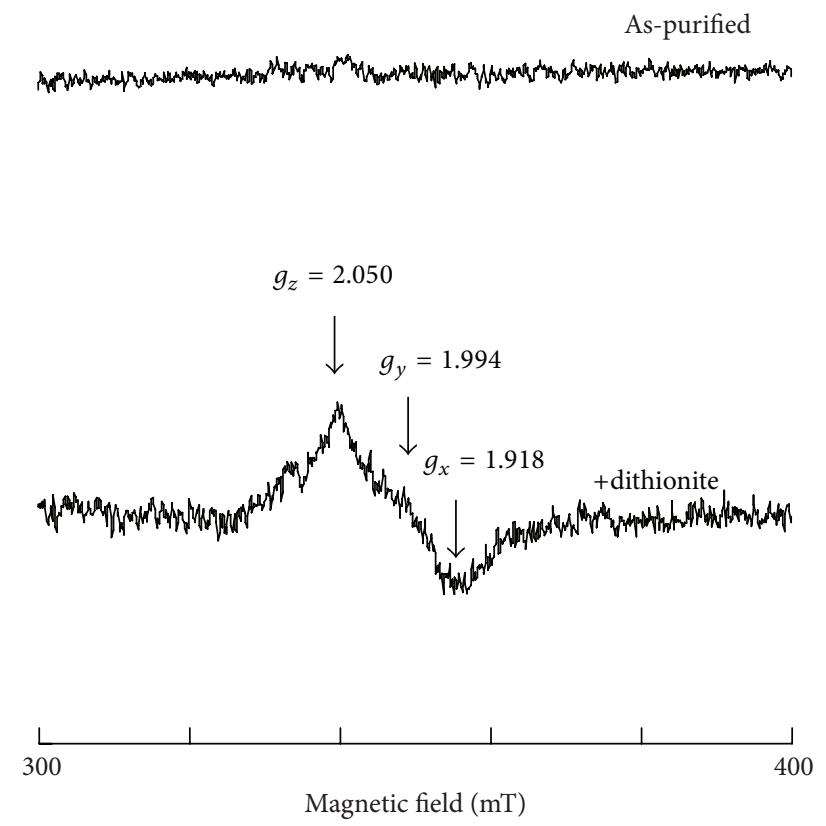

(b)

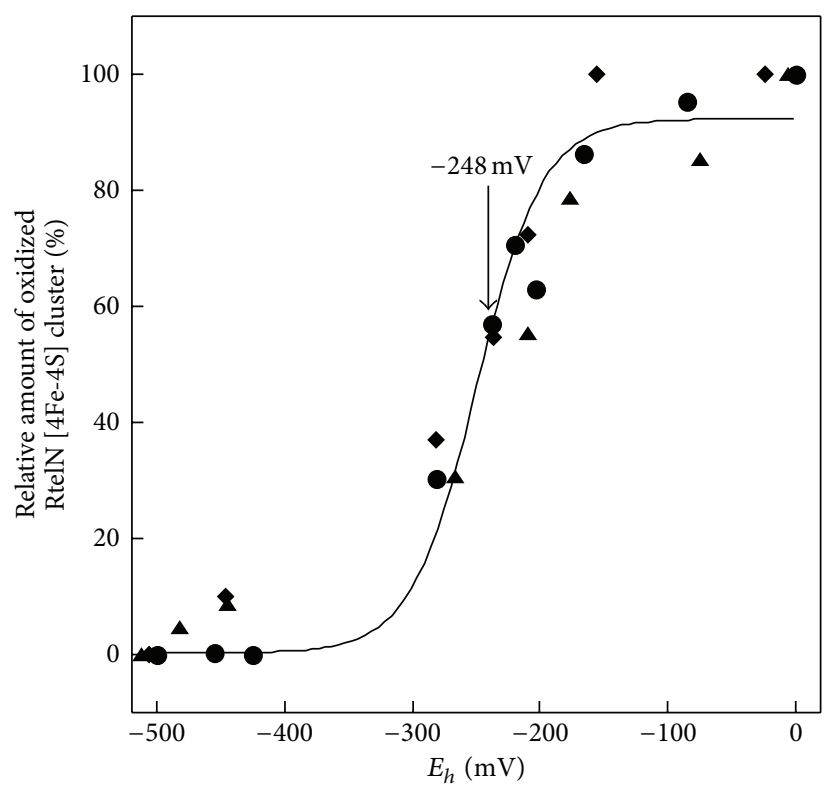

(c)

Figure 3: Redox titration of the RtelN iron-sulfur cluster. (a) UV-visible spectra of purified RtelN. Purified RtelN (40 $\mu \mathrm{M}$ ) (spectrum 1) was reduced with freshly prepared sodium dithionite $(2 \mathrm{mM})$ (spectrum 2). (b) EPR spectra of purified RtelN. RtelN (90 $\mu \mathrm{M})$ (spectrum 1$)$ was reduced with freshly prepared sodium dithionite $(2 \mathrm{mM})$ (spectrum 2). (c) Redox titration of purified RtelN. The amplitudes of the absorbance peak at $415 \mathrm{~nm}$ were normalized to 0 and $100 \%$ for the fully reduced and oxidized RtelN iron-sulfur cluster in solution, respectively. The solid line drawn through three sets of data points represents the best fit to a Nernst equation $(n=1)$ with $E_{m}=-248 \pm 10 \mathrm{mV}$.

(Figure 5), the iron-sulfur cluster could have an important role in other steps of the reaction catalyzed by Rtell.

The telomere length of chromosomes has been linked to intracellular oxidative stress in human cells [38]. The finding that the RtelN iron-sulfur cluster is sensitive to hydrogen peroxide and nitric oxide (Figure 5) may provide a rational explanation for the association between the telomere length of chromosomes and oxidative stress in cells. Since the ironsulfur cluster is essential for the DNA helicase activity of XPD from yeast and archaea [19-22], disruption of iron-sulfur clusters in protein would likely change the helicase activity of Rtell in human cells. If the iron-sulfur cluster in Rtell is as sensitive to hydrogen peroxide or nitric oxide as that in RtelN, the DNA helicase activity of Rtell could be modulated 


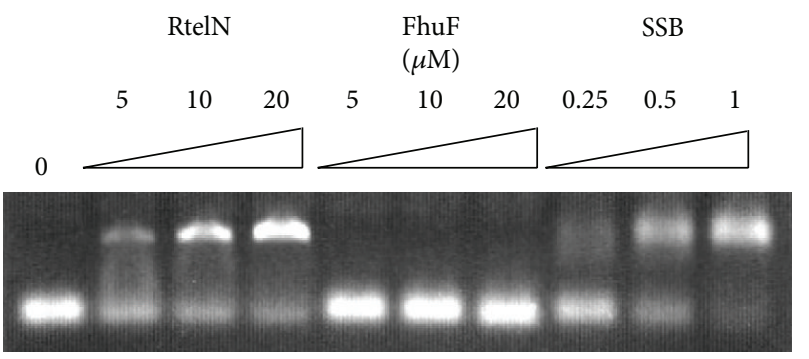

(a)

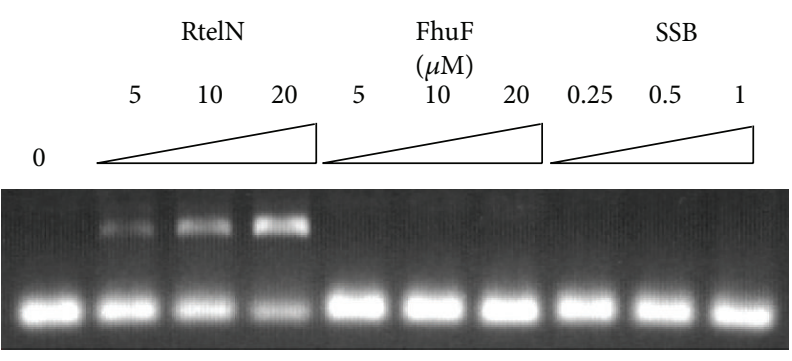

(b)

FIGURE 4: DNA binding activity of purified RtelN. (a) ssDNA binding activity of RtelN. A fluorescence-labeled ssDNA $(0.5 \mu \mathrm{M})$ was incubated with the indicated amount of protein. FhuF is an iron-sulfur protein that has no DNA binding activity. SSB is E. coli ssDNA binding protein. The DNA-protein complex and free DNA probe were resolved on a $0.6 \%$ agarose gel. (b) dsDNA binding activity of RtelN. A fluorescencelabeled dsDNA $(0.5 \mu \mathrm{M})$ was incubated with the indicated amount of protein. The DNA-protein complex and free DNA probe were resolved on a $0.6 \%$ agarose gel. The results are representatives of three independent experiments.

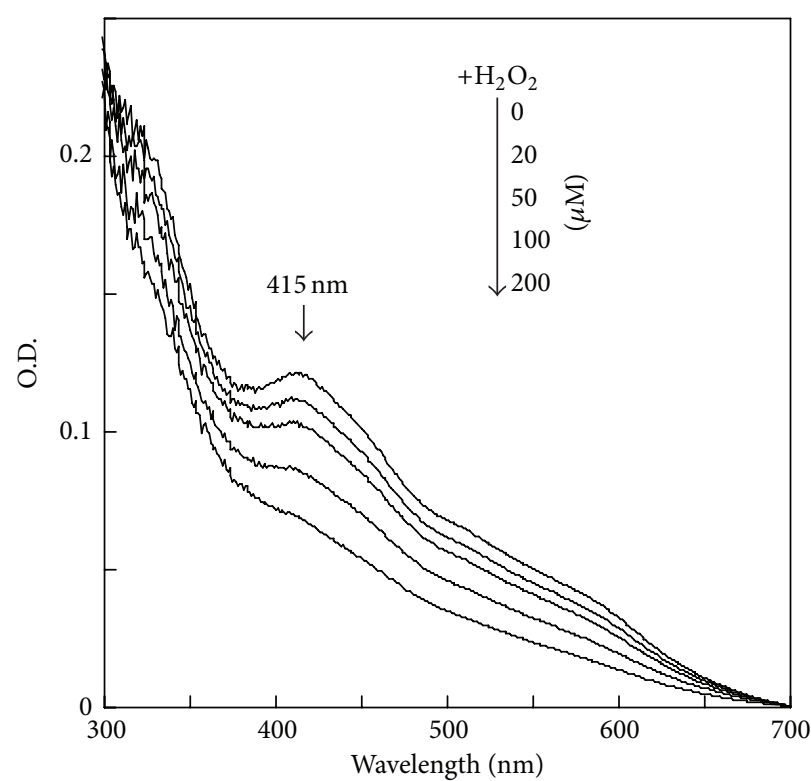

(a)

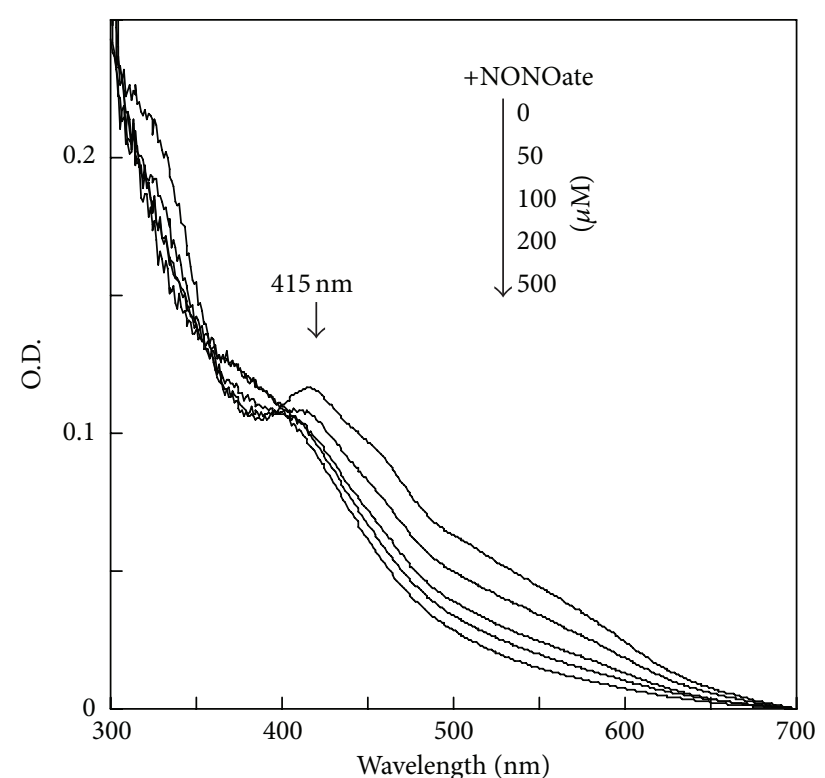

(b)

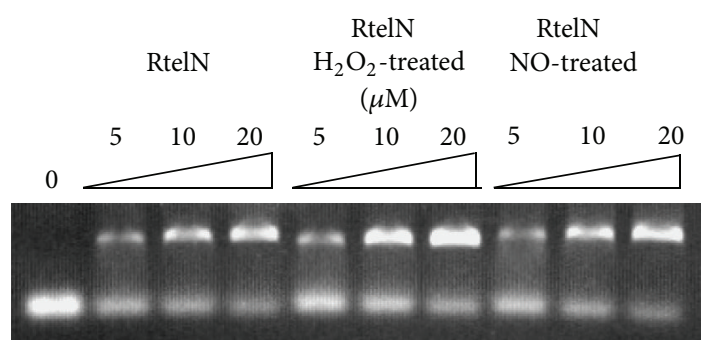

(c)

FIGURE 5: DNA binding activity of RtelN with modified iron-sulfur cluster. (a) Effect of $\mathrm{H}_{2} \mathrm{O}_{2}$ on the RtelN iron-sulfur cluster. RtelN (20 $\mu \mathrm{M}$ ) was incubated with the indicated concentrations of $\mathrm{H}_{2} \mathrm{O}_{2}(0$ to $200 \mu \mathrm{M})$ at room temperature for $30 \mathrm{~min}$. The UV-visible spectra were taken after incubation. (b) Effect of NO on the RtelN iron-sulfur cluster. RtelN $(20 \mu \mathrm{M})$ was incubated with the indicated concentrations of the NO releasing reagent diethylamine NONOate $(0$ to $500 \mu \mathrm{M})$ at $37^{\circ} \mathrm{C}$ for $10 \mathrm{~min}$. The UV-visible spectra were taken after incubation. (c)

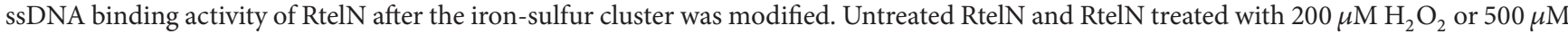
NONOate were incubated with the fluorescence-labeled ssDNA $(0.5 \mu \mathrm{M})$. The DNA-protein complex and free DNA probe were resolved on a $0.6 \%$ agarose gel. The results are representative of three independent experiments. 
by intracellular reactive oxygen/nitrogen species. Therefore, modification of the iron-sulfur cluster in Rtell by reactive oxygen/nitrogen species could at least in part contribute to the telomere length of chromosomes in cells [38]. Evidently, additional experiments are required to illustrate the regulatory role of the iron-sulfur cluster in the human Rtell and other DNA helicases.

\section{Abbreviations \\ EPR: Electron paramagnetic resonance \\ $E_{m}: \quad$ Midpoint redox potential \\ Rtell: Human telomere length regulator \\ RtelN: The N-terminal domain of Rtell \\ ssDNA: Single-stranded DNA \\ dsDNA: Double-stranded DNA.}

\section{Conflict of Interests}

The authors declare that there is no conflict of interests regarding the publication of this paper.

\section{Acknowledgment}

This work was supported in part by the National Cancer Institute of the National Institute of Health Grant CA107494, the American Heart Association Grant 13GRNT16890014 (to $\mathrm{HD}$ ), and the Louisiana Board of Regents Graduate Scholarship (to APL).

\section{References}

[1] T. de Lange, "How telomeres solve the end-protection problem," Science, vol. 326, no. 5955, pp. 948-952, 2009.

[2] A. Sfeir, S. T. Kosiyatrakul, D. Hockemeyer et al., "Mammalian telomeres resemble fragile sites and require TRF1 for efficient replication," Cell, vol. 138, no. 1, pp. 90-103, 2009.

[3] E. H. Blackburn, "Switching and signaling at the telomere," Cell, vol. 106, no. 6, pp. 661-673, 2001.

[4] H. Ding, M. Schertzer, X. Wu et al., "Regulation of murine telomere length by Rtel: an essential gene encoding a helicaselike protein," Cell, vol. 117, no. 7, pp. 873-886, 2004.

[5] Y. Jeffrey Chiang, R. T. Calado, K. S. Hathcock, P. M. Lansdorp, N. S. Young, and R. J. Hodes, "Telomere length is inherited with resetting of the telomere set-point," Proceedings of the National Academy of Sciences of the United States of America, vol. 107, no. 22, pp. 10148-10153, 2010.

[6] E. Uringa, J. L. Youds, K. Lisaingo, P. M. Lansdorp, and S. J. Boulton, "RTEL1: an essential helicase for telomere maintenance and the regulation of homologous recombination," Nucleic Acids Research, vol. 39, no. 5, pp. 1647-1655, 2011.

[7] C. Bai, B. Connolly, M. L. Metzker et al., "Overexpression of M68/DcR3 in human gastrointestinal tract tumors independent of gene amplification and its location in a four-gene cluster," Proceedings of the National Academy of Sciences of the United States of America, vol. 97, no. 3, pp. 1230-1235, 2000.

[8] A. J. Walne, T. Vulliamy, M. Kirwan, V. Plagnol, and I. Dokal, "Constitutional mutations in RTEL1 cause severe dyskeratosis congenita," The American Journal of Human Genetics, vol. 92, no. 3, pp. 448-453, 2013.
[9] B. J. Ballew, M. Yeager, K. Jacobs et al., "Germline mutations of regulator of telomere elongation helicase 1, RTEL1, in Dyskeratosis congenita," Human Genetics, vol. 132, no. 4, pp. 473-480, 2013.

[10] M. E. Fairman-Williams, U. P. Guenther, and E. Jankowsky, "SF1 and SF2 helicases: family matters," Current Opinion in Structural Biology, vol. 20, no. 3, pp. 313-324, 2010.

[11] M. F. White, "Structure, function and evolution of the XPD family of iron-sulfur-containing $5^{\prime} \rightarrow 3^{\prime}$ DNA helicases," Biochemical Society Transactions, vol. 37, no. 3, pp. 547-551, 2009.

[12] S. B. Cantor and S. Guillemette, "Hereditary breast cancer and the BRCA1-associated FANCJ/BACH1/BRIP1," Future Oncology, vol. 7, no. 2, pp. 253-261, 2011.

[13] J. A. Sommers, N. Rawtani, R. Gupta et al., "FANCJ uses its motor ATPase to destabilize protein-DNA complexes, unwind triplexes, and inhibit RAD51 strand exchange," The Journal of Biological Chemistry, vol. 284, no. 12, pp. 7505-7517, 2009.

[14] Y. Wu, J. A. Sommers, I. Khan, J. P. de Winter, and R. M. Brosh Jr., "Biochemical characterization of Warsaw breakage syndrome helicase," Journal of Biological Chemistry, vol. 287, no. 2, pp. 1007-1021, 2012.

[15] J. L. Youds, D. G. Mets, M. J. McIlwraith et al., "RTEL-1 enforces meiotic crossover interference and homeostasis," Science, vol. 327, no. 5970, pp. 1254-1258, 2010.

[16] L. J. Barber, J. L. Youds, J. D. Ward et al., "RTEL1 maintains genomic stability by suppressing homologous recombination," Cell, vol. 135, no. 2, pp. 261-271, 2008.

[17] M. F. White and M. S. Dillingham, "Iron-sulphur clusters in nucleic acid processing enzymes," Current Opinion in Structural Biology, vol. 22, no. 1, pp. 94-100, 2012.

[18] R. A. Pugh, M. Honda, H. Leesley et al., "The iron-containing domain is essential in Rad3 helicases for coupling of ATP hydrolysis to DNA translocation and for targeting the helicase to the single-stranded DNA-double-stranded DNA junction," The Journal of Biological Chemistry, vol.283, no. 3, pp. 1732-1743, 2008.

[19] H. Liu, J. Rudolf, K. A. Johnson et al., "Structure of the DNA Repair Helicase XPD,” Cell, vol. 133, no. 5, pp. 801-812, 2008.

[20] L. Fan, J. O. Fuss, Q. J. Cheng et al., "XPD helicase structures and activities: insights into the cancer and aging phenotypes from XPD mutations," Cell, vol. 133, no. 5, pp. 789-800, 2008.

[21] S. C. Wolski, J. Kuper, P. Hänzelmann et al., "Crystal structure of the FeS cluster-containing nucleotide excision repair helicase XPD," PLoS Biology, vol. 6, article e149, no. 6, 2008.

[22] J. Rudolf, V. Makrantoni, W. J. Ingledew, M. J. R. Stark, and M. F. White, "The DNA repair helicases XPD and FancJ have essential iron-sulfur domains," Molecular Cell, vol. 23, no. 6, pp. 801-808, 2006.

[23] B. Ren, X. Duan, and H. Ding, "Redox control of the DNA damage-inducible protein DinG helicase activity via its ironsulfur cluster," Journal of Biological Chemistry, vol. 284, no. 8, pp. 4829-4835, 2009.

[24] J. T. P. Yeeles, R. Cammack, and M. S. Dillingham, "An ironsulfur cluster is essential for the binding of broken DNA by AddAB-type helicase-nucleases," The Journal of Biological Chemistry, vol. 284, no. 12, pp. 7746-7755, 2009.

[25] R. E. Cowart, F. L. Singleton, and J. S. Hind, "A comparison of bathophenanthrolinedisulfonic acid and ferrozine as chelators of iron(II) in reduction reactions," Analytical Biochemistry, vol. 211, no. 1, pp. 151-155, 1993. 
[26] L. M. Siegel, "A direct microdetermination for sulfide," Analytical Biochemistry, vol. 11, no. 1, pp. 126-132, 1965.

[27] R. D. Shereda, A. G. Kozlov, T. M. Lohman, M. M. Cox, and J. L. Keck, "SSB as an organizer/mobilizer of genome maintenance complexes," Critical Reviews in Biochemistry and Molecular Biology, vol. 43, no. 5, pp. 289-318, 2008.

[28] Z. Cheng, A. Caillet, B. Ren, and H. Ding, "Stimulation of Escherichia coli DNA damage inducible DNA helicase DinG by the single-stranded DNA binding protein SSB," FEBS Letters, vol. 586, no. 21, pp. 3825-3830, 2012.

[29] P. Leslie Dutton, "Redox potentiometry: determination of midpoint potentials of oxidation-reduction components of biological electron-transfer systems," Methods in Enzymology, vol. 54, pp. 411-435, 1978.

[30] G. Böhm, R. Muhr, and R. Jaenicke, "Quantitative analysis of protein far UV circular dichroism spectra by neural networks," Protein Engineering, vol. 5, no. 3, pp. 191-195, 1992.

[31] K. Müller, B. F. Matzanke, V. Schünemann, A. X. Trautwein, and K. Hantke, "FhuF, an iron-regulated protein of Escherichia coli with a new type of [2Fe-2S] center," European Journal of Biochemistry, vol. 258, no. 3, pp. 1001-1008, 1998.

[32] S. Jang and J. A. Imlay, "Micromolar intracellular hydrogen peroxide disrupts metabolism by damaging iron-sulfur enzymes," The Journal of Biological Chemistry, vol. 282, no. 2, pp. 929-937, 2007.

[33] A. P. Landry, X. Duan, H. Huang, and H. Ding, "Iron-sulfur proteins are the major source of protein-bound dinitrosyl iron complexes formed in Escherichia coli cells under nitric oxide stress," Free Radical Biology and Medicine, vol. 50, no. 11, pp. 1582-1590, 2011.

[34] D. R. Hyduke, L. R. Jarboe, L. M. Tran, K. J. Y. Chou, and J. C. Liao, "Integrated network analysis identifies nitric oxide response networks and dihydroxyacid dehydratase as a crucial target in Escherichia coli," Proceedings of the National Academy of Sciences of the United States of America, vol. 104, no. 20, pp. 8484-8489, 2007.

[35] Y. Wu and R. M. Brosh Jr., "DNA helicase and helicase-nuclease enzymes with a conserved iron-sulfur cluster," Nucleic Acids Research, vol. 40, no. 10, pp. 4247-4260, 2012.

[36] C. T. Dooley, T. M. Dore, G. T. Hanson, W. C. Jackson, S. J. Remington, and R. Y. Tsien, "Imaging dynamic redox changes in mammalian cells with green fluorescent protein indicators," Journal of Biological Chemistry, vol. 279, no. 21, pp. 2228422293, 2004.

[37] J. M. Hansen, Y. M. Go, and D. P. Jones, "Nuclear and mitochondrial compartmentation of oxidative stress and redox signaling," Annual Review of Pharmacology and Toxicology, vol. 46, pp. 215-234, 2006.

[38] J. M. J. Houben, H. J. J. Moonen, F. J. van Schooten, and G. J. Hageman, "Telomere length assessment: biomarker of chronic oxidative stress?" Free Radical Biology and Medicine, vol. 44, no. 3, pp. 235-246, 2008. 

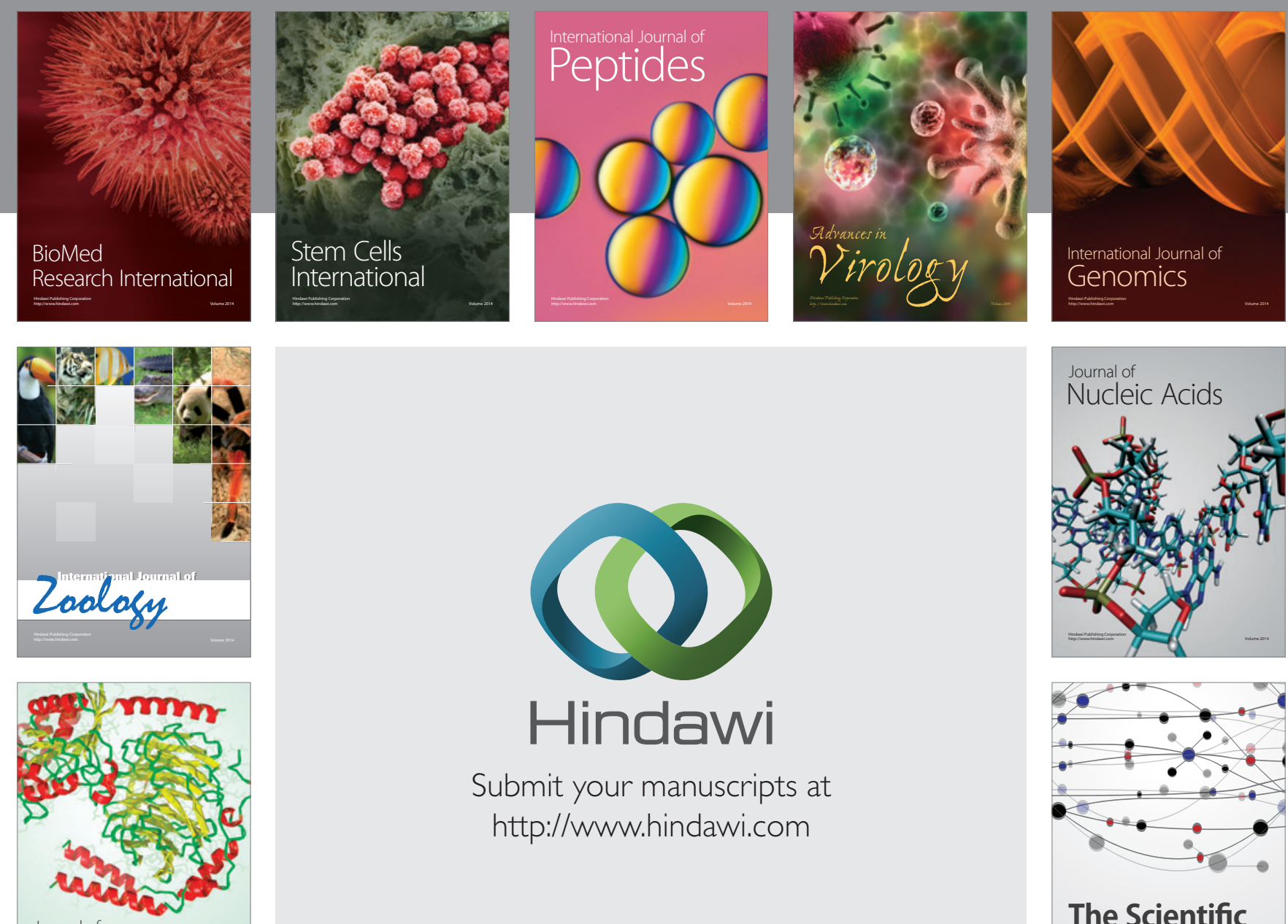

Submit your manuscripts at

http://www.hindawi.com

Journal of
Signal Transduction
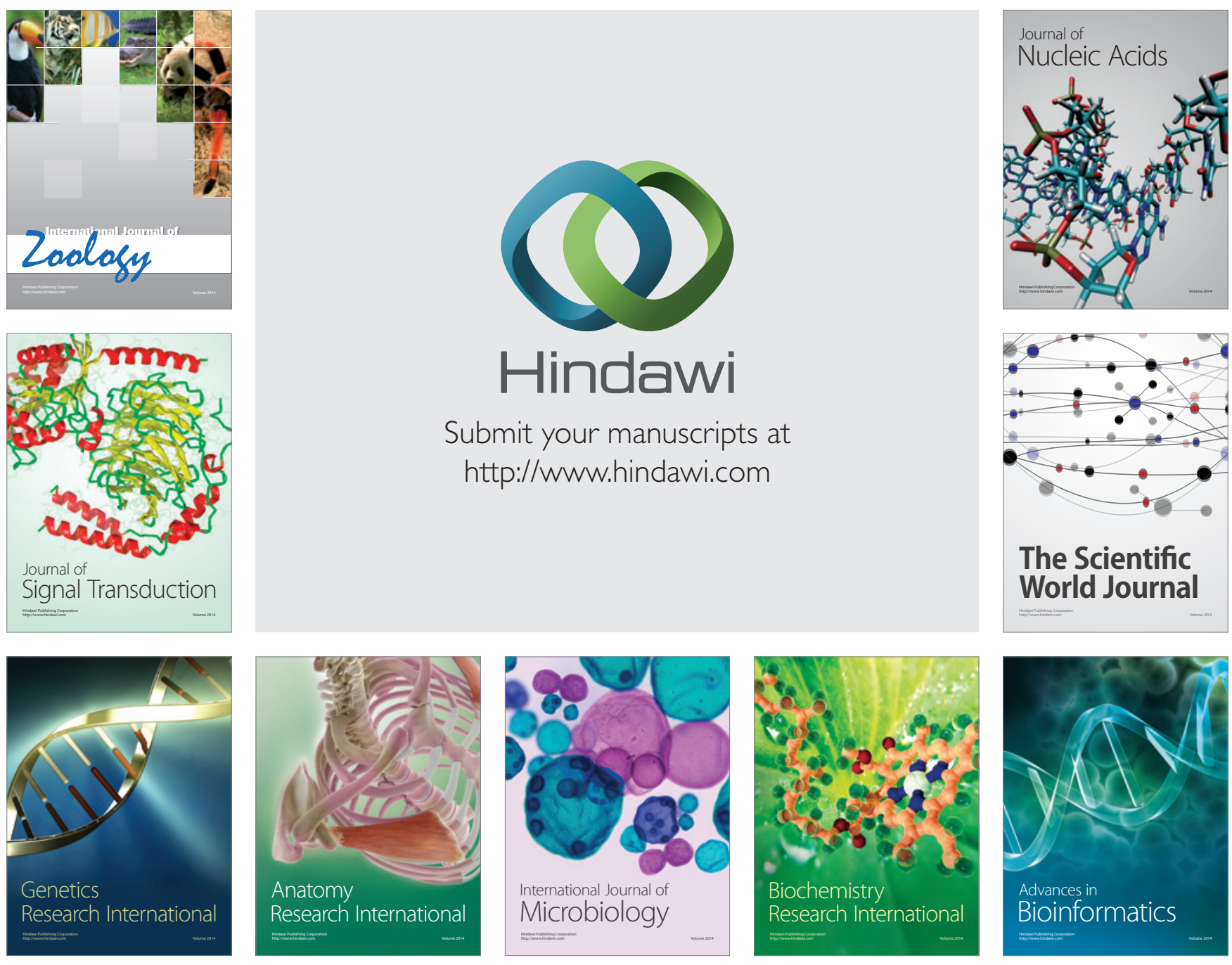

The Scientific World Journal
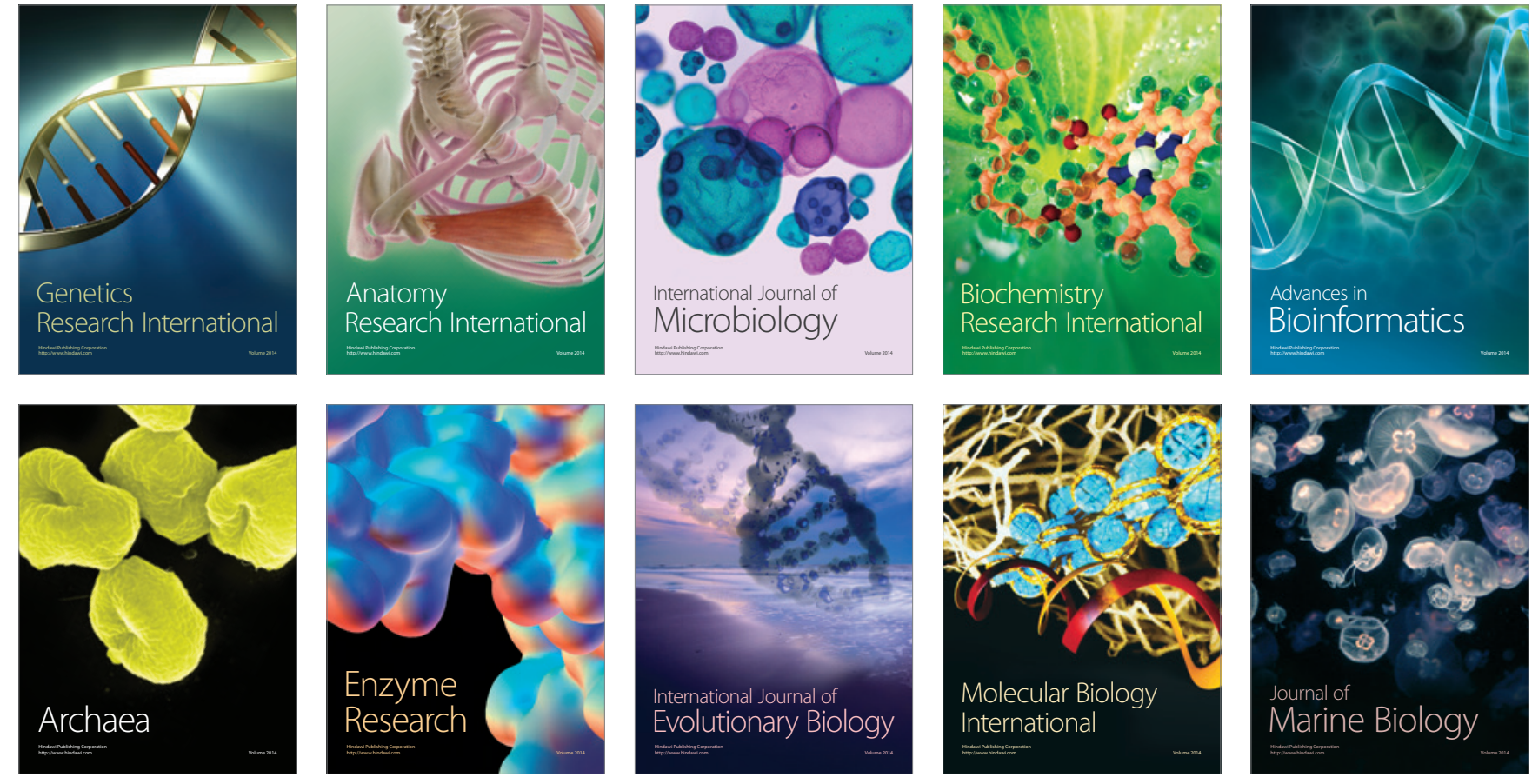